\title{
Ethical Philanthropy in the era of Patient-Centric, Value-Based Health Care
}

\author{
Peter Kalina \\ MD, MBA, FACR \\ Department of Radiology \\ Mayo Clinic, 200 First Street, Rochester, MN 55902
}

\begin{abstract}
Patient-centered, value-based health care implies care that is high-quality, responsive to patient preferences, delivered safely, and at a fair cost. The future of healthcare portends changes; including less favorable payer mix, decreased reimbursement and alternate compensation models. Innovative strategies will be required to maintain excellence in care while efficiently maximizing existing capital and human resources. Successfully implementing a health care institution's initiatives cannot be achieved entirely from within. Philanthropy must fill gaps to help support research, education and clinical missions. Strong physician - patient relationships are essential to facilitating philanthropic gifts from grateful patients. While we assume magnanimous intentions and motivations, gifts may potentially influence behavior. While VIP care for patients who donated (or believed likely to) is a successful strategy; distinctions are needed between development and clinical relationships. Philanthropy cannot affect care, which must be irrespective of wealth or position. The highest professional and ethical standards and practices must be maintained when accepting grateful patient philanthropy. Developing and promoting a sustainable philanthropic strategy begins with reviewing needs and goals to support the mission; motivated and inspired by improving outcomes. Achieving missions in health care requires dedicated philanthropic partnerships. Pursued with professionalism, thoughtfulness and integrity; philanthropy represents a vital pathway to innovatively advance patient care. Support allows donors to make an investment and express their values. Realizing the direct and lasting impact of collaboration provides rewarding benefit and meaningful recognition.
\end{abstract}

\section{INTRODUCTION}

"Patient-centric, value-based health care" is much more than a ubiquitous marketing slogan. It describes consistently high-quality care, delivered by world-class providers, utilizing state-ofthe-art equipment, guided by practical performance and process improvement parameters. It requires an expectation of the utmost in safety throughout all interactions within the system. It presumes timely access, ease of appointments, open communication, user-friendly parking and the many other "radical conveniences" today's patients expect; and deserve. It means these parameters are delivered at a fair cost. Value = good outcomes = quality, safety, service, patient satisfaction .......at a low cost. Patient-centered = providing care that is responsive to patient preferences so that patient values guide clinical decisions.

The economic climate of healthcare will continue to change. The likelihood exists for a less favorable payor mix, decreased reimbursements, and alternate payment/compensation models (bundled care, pay-for-performance, etc) supplanting fee-for-service and productivitybased incentives. Innovative strategies will be required to maintain our priority on excellence in patient care. As stewards, we will need to be responsive to change, and efficient at maximizing our existing capital and human resources - "do more with less." Yet, this must never be at the expense of quality and safety. More frequent encounters with PA's and NP's 
will help address cost and access issues, providing opportunities for families to optimize their health locally, in a convenient and more cost-effective manner.

\section{PHILANTHROPY}

The successful implementation of a health care institution's myriad bold initiatives cannot be achieved entirely from within. The past decade has seen reductions in federal funding for medical research, along with constraints on state and local budgets for clinical programs [Ohman 2018, Collins 2018(a)]. This creates tremendous challenges for medical schools, academic medical centers (AMC), and healthcare systems. Many institutions have large gaps that philanthropy must fill in order to help support and facilitate the necessary research, education and clinical missions. In 2017, health care institutions in the US received $\$ 10.1$ billion in charitable gifts from individuals and foundations. Of that, approximately 20\% of philanthropic support to AMC's was from grateful patients who recognized the excellent care they had received and the meaningful relationships that were established [Collins 2018(b)]. Private individual and foundation gifts rival or surpass federal grants given the greater availability of philanthropic dollars coinciding with the decline in NIH grants. Philanthropic funding can accelerate progress by being more nimble, given that private donors don't have to answer to voters or shareholders (Wheeler 2014).

\section{COMMUNITY}

Large health care organizations and systems must think broadly. Transformational impact on a national and global scale is of vital importance. Yet, so too is facilitating and maintaining close excellent relationships with their local communities. Utilizing community health needs assessments (CHNA) help delineate a pattern of potential beneficial impact on both individual and population health. Community based programs contribute greatly to a strong, supported, and supportive community. This, in turn, lays a solid foundation upon which to build.

\section{THE DEVELOPMENT TEAM}

In any industry, the most important attribute portending success is one's employees. In health care, employees entrusted to engage patients must be armed with a clear and profound knowledge and understanding of the organizations culture, mission, vision and compelling goals. These employees are empowered to execute that vision via well-planned strategies. Recruiting, retaining and developing a talented, skilled and dedicated high performing team is critical to success; requiring development and maintenance of close relationships.

Many of us are extremely fortunate to be employees and stewards of highly trusted, worldclass health care institutions. The reputations garnered are well deserved; earned through a strong work ethic, consistency, transparency, honesty, and integrity. Of all the potential attributes vital to a successful development initiative, perhaps none is more critical than a culture of trust. Not-for-profit hospitals and healthcare organizations are understandably considered among the most trusted entities. We must maintain this vital public trust. Executing the ambitious and robust capital project, patient care, education and research goals at the core of the mission and vision of modern health care facilities will be more challenging in the future. Every opportunity must be taken to continually strengthen and build upon existing status and trust.

Employees committed to their purpose of making a meaningful positive impact on the lives of patients are vital to success. Their passion will naturally and seamlessly be reflected in their interactions with patients and with each other. Caring, empathy and enthusiasm will permeate all that they do. Motivation for the success of the mission serves to create strong ambassadors for the institution. These are the people at "the front lines," interacting with patients on a daily 
basis. Like the superbly trained staff at the reception desk of every Four Seasons Hotel around the world, these are the individuals paramount to maintaining and expanding your brand and your reputation (Kalina, 2018).

Consistent outstanding care and service means a reproducible, positive, memorable experience. Exceptional customer service by proud, passionate, professional and allied health staff stems from anticipating and exceeding "customer "expectations. If the work environment includes a culture of trust, honesty, respect, integrity, fairness and transparency; a motivated team will commit to helping deliver consistent, world-class care and patient satisfaction.

In addition to drive, dedication and experience, individuals and teams successful in development leadership roles must possess a wide range of attributes, competencies and "hard skills." Of the "soft skills," perhaps none is more important than the personality to comfortably and confidently engage the diverse population of unique individuals seeking to help you support your mission. The development team must genuinely enjoy wide-ranging interactions and conversations, in both formal and informal settings. We live in a digital age where social media rules. However, there remains a need for hand-written notes; a time for heartfelt telephone calls. Sometimes, there is simply no substitute for warm personal visits. Communication skills are vital, and should be tailored to each individual. Each encounter will be with someone whose unique interests and specific preferences will address why and how they wish to be involved as a philanthropic partner in helping you achieve your mission, vision and goals.

The functions of an effective and successful development team will dramatically impact the operations, strategic planning, implementation, stability and growth of a health care institution. A cadre of enthusiastic team members and key stakeholders will work together contributing to identify opportunities, through consensus and aligned priorities, toward a common and compelling vision. A coordinated effort will determine how to proceed with initiatives, maintaining existing pipelines, and exploring the creation of new prospective partnerships. Collaborating to develop, build and strengthen meaningful and lasting personalized relationships should be a primary goal.

\section{STRATEGIES}

Organizations rely on metrics; defining, measuring, analyzing, improving, controlling and scrutinizing everything detail deemed critical to achieving their benchmarks, target and mission. Describing the accomplishments and needs of your organization and its dedicated employees should be based on specifics, facts and objective criteria. Data should drive your strategy and why you choose a compelling course of action. "Why" is the most crucial question to ask. Why are we doing something? Follow that up with determining "what" to do, and "how" to implement your proposed strategy; along with what you will say publicly.

Given that the future of health care will, by all accounts, look very different; "disruptive innovation" is needed. Maintaining the status quo will not be adequate. A culture of teamwork, collaboration and aligned goals assures excellent patient outcomes. "Radical inclusion" means seeking out advice, perspective and contributions from a broad range of determined and diverse individuals. This must include like-minded and more importantly, those with dissenting viewpoints and opinions. Seek out the input, ideas and expertise of a strategically developed team. Sharing insights, seeking feedback, discussing best practices, overcoming challenges, and debating differences of opinion will allow us to work together cohesively to optimize creativity and innovation. 
The Institute for Healthcare Improvement (IHI) describes the optimization of health system performance as four simultaneous mutually reinforcing components; the "quadruple aim" a) improving the patient care experience (quality and satisfaction), b) improving population health, c) reducing costs and d) enhancing provider well-being. While patient outcomes are paramount, providers must be physically and emotionally healthy in order to optimally care for patients. Threats to quality, safety, engagement and patient satisfaction contribute to a compelling societal and business case for organizations to monitor and manage physician burnout. Physicians' desire to shape their work to be meaningful and fulfilling is threatened by increased workloads and by work-flow that is standardized to increase throughput and consistency, while reducing waste, variability and risk. Combined patient and provider satisfaction confer optimal outcomes, a competitive business advantage, and an improved financial bottom-line.

\section{ETHICAL CONSIDERATIONS}

While strong physician - patient relationships are essential to facilitating philanthropic gifts from grateful patients, potential ethical concerns and challenges may arise. We assume magnanimous intentions and motivations of patient giving - they represent a show of gratitude. Although perhaps not intentionally, personal gifts from patients to physicians or to institutions have the potential to influence behavior; and expectations. Are we more attentive and responsive to benefactors? Do they get better access? Do we "squeeze" them in to an otherwise full schedule? Does a development officer escort them to their next appointment? No waiting?

These benefits of "VIP" or "concierge" care for patients who've donated or believed likely to, portend a successful strategy for cultivating gifts (Wright 2013). However, we must proceed carefully. Clear distinctions are needed between development work and the primary clinical relationship with the patient. Philanthropy cannot affect the care provided to patients, which must be considered irrespective of their wealth, position, power or influence (Ohman 2016). Avoid direct solicitation of ones' own patients, especially at the time of the clinical encounter (Wright 2013, Prokopetz 2014). Delegate the cultivation and solicitation of financial gifts to development professionals.

Maintain dedication to the highest professional and ethical standards and practices when accepting grateful patient philanthropy. While honoring donors' intentions, safeguard the integrity of all that is underwritten by philanthropy (Wheeler 2014). As responsible and trusted stewards, be prepared to turn down any gift with the potential to violate ethical principles.

\section{CONCLUSIONS}

Developing and promoting an ambitious and sustainable philanthropic strategy begins with reviewing current and future needs to support the mission. The motivation and inspiration for your strategies; the driver of your operations; is improving patient-centric, value-driven outcomes. A passion for compelling core values represents the ultimate competitive advantage. A culture of quality, safety, optimal patient experience and "customer" satisfaction ("the needs of the patient come first") guide the efforts. Patients expect, and deserve specific positive outcomes, along with "radical conveniences." Identifying new opportunities to improve the patient experience in meaningful ways assures maximizing benefits for all stakeholders (Wheeler 2014). The goal in today's health care environment is to enhance efficiency, productivity, cost-effectiveness and profitability; while assuring that sustainable process management systems maintain autonomy, flexibility, creativity and innovation. 
Our missions in health care are bold and meaningful. Achieving them requires passion along with dedicated relationships and partnerships. They require the engagement, guidance, generosity and contribution of time and resources from visionaries whose intentions are aligned with the institutional mission. When pursued with professionalism, accountability and integrity; philanthropy represents a vital pathway to innovatively advancing patient care.

Supporting an institution allows donors to make an investment; an opportunity to express specific personal interests, values and desires to make a significant and measurable impact. At the same time, they are partners in advancing the mission of the institution and stimulating innovation. Health care is big business requiring protecting and promoting the brand; optimizing financial performance by meeting or exceeding metrics, benchmarks and targets; and developing and executing strategies for new opportunities. For our magnanimous and altruistic partners, the empowerment and realization of the direct and lasting impact of their collaboration will be their most rewarding benefit, and most meaningful acknowledgement and recognition.

\section{References}

Collins M, Rum S, Sugarman J. 2018(a) Navigating the Ethical Boundaries of Grateful Patient Fundraising. JAMA. 320(10):975-976

Collins M, Rum S, Wheeler J et al. 2018(b) Ethical Issues and Recommendations in Grateful Patient Fundraising and Philanthropy. Acad Med. 93:1631-1637

Institute for Healthcare Improvement. Triple Aim: better care for individuals, better health for populations and lower per capita costs. Available at: http://www.ihi.org/engage/initiatives/tripleaim/pages/default.aspx. Accessed 5/25/19

Kalina P. (2018) Strategic Healthcare Marketing. What can we learn from the hospitality industry. Human Resource Management Research 8(2):42-44

Ohman E, Douglas P, Dean L, Ginsburg G. 2016 Philanthropy for Science: Is It a Viable Option? Circulation Research. 119:1057-1059

Prokopetz J, Lehmann L. (2014) Physicians as fundraisers: medical philanthropy and the doctor-patient relationship. PLOS Medicine 11(2):1-3

Wheeler J, Rum S, Wright S. (2014) Philanthropy, Medical Research, and the Role of Development. The American Journal of Medicine. 127(10):903-904

Wright S, Wolfe L, Stewart R, et al. (2013) Ethical Concerns Related to Grateful Patient Philanthropy: The

Physician's Perspective. J Gen Intern Med 28(5):645-51 